Research Article

\title{
Simulation Investigation of the Stowing and Deployment Processes of a Self-Deployable Sunshield
}

\author{
Xu Cao $\mathbb{D}^{1,2}$ Yan Xu $\mathbb{D}^{3}$, Changhong Jiang $\mathbb{D}^{1},{ }^{1}$ Qin Fang $\mathbb{D}^{3}$, and Hao Feng $\mathbb{}^{1}$ \\ ${ }^{1}$ Beijing Institute of Space Mechanics and Electricity, Beijing 100094, China \\ ${ }^{2}$ School of Aerospace Engineering, Beijing Institute of Technology, Beijing 100081, China \\ ${ }^{3}$ School of Aeronautics and Astronautics, Zhejiang University, Hangzhou 310027, China \\ Correspondence should be addressed to Yan Xu; xyzs@zju.edu.cn
}

Received 4 November 2020; Revised 11 December 2020; Accepted 4 January 2021; Published 8 February 2021

Academic Editor: Antonio Concilio

Copyright ( $\odot 2021 \mathrm{Xu}$ Cao et al. This is an open access article distributed under the Creative Commons Attribution License, which permits unrestricted use, distribution, and reproduction in any medium, provided the original work is properly cited.

The stowing and deployment processes of a self-deployable sunshield are investigated numerically in this paper. The composition of the self-deployable sunshield is described. Deployed moment theoretical models for lenticular booms are formulated based on the bending theory of curved shell. The numerical analysis method of deployed moment is proposed. Two types of control methods for a fold crease are presented, and a dynamic analysis model considering geometry and nonlinear contact is built. The analysis results indicate that the press flattening method can be used effectively for controlling the fold crease, and the analytical results of the deployed moment are very close to the theoretical results. A stowing and deployment process analysis of the selfdeployable sunshield is conducted. Thus, the deployment configurations and the time history curves of the dynamic behaviors are obtained. The results verify the feasibility of the analysis model, and this study can provide technical support for the engineering application of the self-deployable sunshield.

\section{Introduction}

The optical system of a spacecraft, especially the optical telescope, needs to be designed with a sunshield to suppress the influence of external stray light. At the same time, a stable thermal environment must be provided for spacecraft structures to prevent excessive thermal deformation. For large spacecraft structures, due to the constraints of launch volume and weight, sunshields must be designed as deployable structures. Initially, these deployable sunshields are stowed in a folded state and deployed upon achieving orbit.

The structural styles of these deployable sunshields are mainly planar and cylindrical, while the deployment mechanisms include inflatable, mechanical, and elastic deployments. Open planar deployable sunshields have been frequently adopted when the spacecraft's optical system can undergo passive self-cooling. For example, the sunshields for the second-generation Hubble telescope (NGST) [1] were designed with four in-plane, cross-shaped, inflatable tubes to deploy skin films for the sunshields. Another representative project was the sunshields on the James Webb space tele- scope (JWST) [2, 3], which was made up of five layers of flexible film that can be deployed. By means of a series of miniature springs, the folded film was controlled to deploy layer by layer. Cylindrical sunshields are usually placed around the optical system circumferentially. One end is fixed to the spacecraft structures, and the other end is deployed at the far end of the optical system. A cylindrical sunshield was proposed for the International X-ray Observatory (IXO) [4], which is approximately 10 meters high and 4 meters in diameter. There were two kinds of deployable mechanism design schemes: the first one used a deployable linkage with an active hinge in the middle of the rod, which can extend for approximately $20 \mathrm{~m}$. The other mechanism was driven by three deployable arms, which were approximately $24 \mathrm{~m}$ in height after full deployment, and the structure diameter did not change. The sunshield of MITAR [5], which was supported by Italian Space Agency (ASI), has an octagonal frame structure. Elastic spring tapes were used to pull the structure upon achieving orbit. A cone sunshield CSS [6] was divided into six hood boards supported by inflatable vertical and horizontal beams. Most of these approximate cylindrical 
sunshields can only be deployable in the axial direction, and the size of the radial direction is invariable. The abovementioned research and development efforts mainly focus on mechanism designs of deployable sunshields, and few detailed studies of mechanical characteristics are involved.

Up to now, the dynamic and wave characteristics of shell structures have been studied extensively. In the field of vibration analysis in the shell, Allam et al. provided an analytical model based on a generalized and simple refined higherorder shear deformation theory to analyze fundamental frequencies of simply supported laminated composite/sandwich shells [7]. Khadimallah et al. studied the analytical rotating vibration for functionally graded shell with ring supports [8]. The fundamental natural frequency of cylindrical shell of parameter versus ratios of length- and height-to-radius for a wide range has been investigated [9]. Cuong-Lea et al. investigated the free vibration and buckling responses of annular plate, conical, and cylindrical shell by isogeometric analysis (IGA) method [10]. In the field of elastic wave characteristics, characteristics of the propagated wave in a sandwich shells are investigated by employing Hamilton's principle [11]. Behrouz et al. studied elastic bulk wave characteristics of doubly curved nanoshell [12].

There are few studies on space thin-walled structures such as lenticular boom. The European Space Agency (ESA) and the German Aerospace Center (DLR) have conducted extensive research on the lightweight design and deployment mechanisms of flexible thin-walled tubes. The DLR has developed a large thin-walled CFRP tube for space arms [13]. The section shape and drive system were designed so as to realize the deployable support structure of the membrane antenna and solar sail. There have been several studies on the lenticular boom and its unfolding process. Sickinger et al. used explicit analysis software to analyze the stiffness and buckling stability of a lenticular boom, and the thermal/vacuum development tests were carried out [14]. The dynamic behaviors of the whole structures supported by lenticular booms were not investigated.

One of the key technologies implemented in deployable structures is that of a foldable and expandable system. Detailed numerical investigations of the folding patterns and deployment mechanisms of these structures have been necessary. There is a large amount of published literature on deployment simulations and research studies on these deployable structures to date. PAM-CRASH software was used to simulate the deployment of an inflatable structure [15]. Folding patterns, parametric model methods, and software simulations of the deployment mechanisms of inflatable antenna structures were then investigated in detail [16]. Using the finite particle method (FPM), a motion analysis approach of mechanically deployable structures based on a straight-rod hinge and angulated-rod hinge was presented [17]. A self-deployable structure with flexible joints was investigated [18]. The method was applied to a spatial system in an analysis that used static equilibrium and kinematic deployment simulations. However, few studies have been performed on the folded and stowed mechanisms of deployable structures. The folded state is mostly obtained by geometric modeling software or parametric modeling. To investigate the folded state and dynamic characteristics accurately, it is necessary to study the entire stowing and deployment processes of deployable structures.

This paper reports on a recently conducted effort that developed an explicit analysis method for stowing and deployment processes of a self-deployable sunshield, which was successfully deployed in orbit. The deployed moments of the lenticular booms are analyzed by theoretical model and numerical simulation model. The paper is organized as follows: Section 2 mainly describes the composition of the self-deployable sunshield, including the structural members, geometric dimensions, and material design. The deployed moment theoretical models for lenticular booms are formulated based on a shell bending theory in Section 3. Section 4 presents a numerical analysis method of the deployed moment, including the fold crease control methods and the dynamic model method. Section 5 establishes a stowing and deployment process analysis model of the self-deployable sunshield. Finally, Section 6 summarizes the concluding remarks and offers suggestions for future works.

\section{Self-Deployable Sunshield Based on Lenticular Boom}

To shield a camera on a satellite platform, a self-deployable sunshield is designed with a metal lenticular boom as the deployment driving structure and a multilayer composite film as the skin structure. The sunshield can be repeatedly folded and self-deployed without external energy. The total weight of the sunshield is $2.7 \mathrm{~kg}$, with a folding ratio of $1: 6$. In November 2019, self-deployable sunshields were successfully deployed in orbit. After the sunshield was deployed, the camera and sunshield will work together while in orbit for 8 years.

The self-deployable sunshield is made up of three separate flat sunshield plates, each of which is supported by two lenticular booms. The folded state and deployed state of the self-deployable sunshield are shown in Figure 1. The length and width of each flat sunshield plate are $610 \mathrm{~mm}$ and $350 \mathrm{~mm}$, respectively. The lenticular boom is composed of two similar beryllium bronze pieces, the length of which is $610 \mathrm{~mm}$, and the wall thickness is only $0.13 \mathrm{~mm}$. The elastic modulus of beryllium bronze is $120 \mathrm{GPa}$, and Poisson's ratio is 0.35 . To ensure a high reliability of deployment, a number of reinforcement pieces were added near the fold crease of the lenticular booms, the material of which is also beryllium bronze. The section shape and sizes of the reinforcement pieces are the same as those of the lenticular boom. The length is $250 \mathrm{~mm}$, and the thickness is $0.15 \mathrm{~mm}$. The number of upper and lower reinforcement pieces on the two sides of the sunshield plate is 2 and 6 , and the number of upper and lower enhancement pieces on the middle sunshield plate is 1 and 5 . The spacing between the reinforcing piece and the beryllium bronze piece and between the adjacent reinforcing pieces is $0.15 \mathrm{~mm}$. Since the three flat sunshield plates are independent of each other, the stowing and deployment processes of the side sunshield plate are investigated in this paper only. 


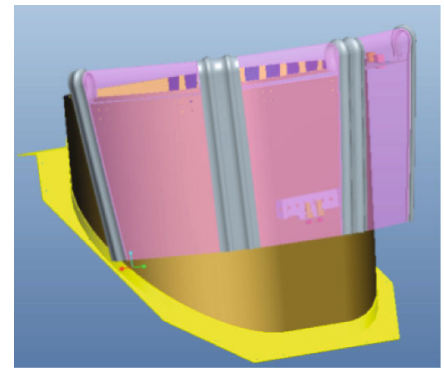

(a) Folded state

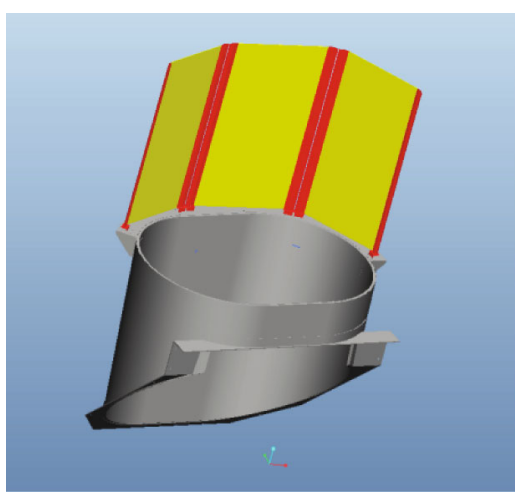

(b) Deployed state

FIgURE 1: Self-deployable sunshield.
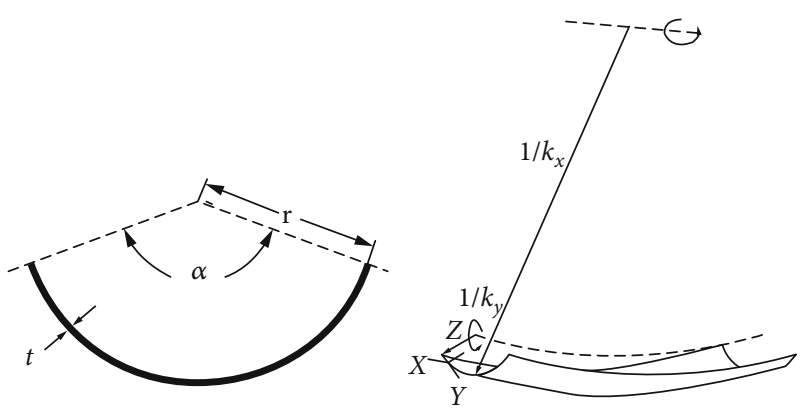

Figure 2: The section and the curvatures of beryllium bronze pieces.

The material of skin structures is designed as a multilayer composite film with a thickness of only $0.25 \mathrm{~mm}$. The surface density of the multilayer composite film is not greater than $300 \mathrm{~g} / \mathrm{m}^{2}$. The skin structures consist of five functional layers, of which the inner surface facing the camera is a black polyimide film with a surface absorption rate of more than 0.8 . The outer layer is made of an extremely thin aluminum foil layer to resist atomic oxygen erosion, and the middle layer is made of polyimide fabric as the bearing layer. Silicone rubber is used as a bonding compound between the three layers. A connecting sleeve is installed on the skin structures for connecting the skin with each lenticular boom, and the lenticular booms are threaded into the connecting sleeve.

\section{Deployed Moment for Lenticular Booms}

3.1. Deployed Moment of Beryllium Bronze Pieces. To reevaluate the deployed performance of lenticular booms, the deployed moment of beryllium bronze pieces is theoretically deduced as follows. According to the bending theory of the thin curve shell based on elastic mechanics $[19,20]$, there are

$$
\left.\begin{array}{l}
M_{x}=D\left(\Delta k_{x}+\mu \Delta k_{y}\right) \\
M_{y}=D\left(\Delta k_{y}+\mu \Delta k_{x}\right)
\end{array}\right\},
$$

where $D$ is the bending stiffness of the thin shell, $D=E t^{3} / 12$ $\left(1-\mu^{2}\right), E$ is the modulus of elasticity of beryllium bronze, $\mu$ is Poisson's ratio, and $\Delta k_{x}$ and $\Delta k_{y}$ are the curvature changes in the two directions, as shown in Figure 2.

Assume that the curvature of the cross-section changes from $1 / \mathrm{R}$ to 0 when the shell is bending, and the curvature along the length changes from 0 to $1 / R$, as shown in Figure 3 .

Then,

$$
\begin{aligned}
\Delta k_{x} & =\mp \frac{1}{r}, \\
\Delta k_{y} & =\frac{1}{R} .
\end{aligned}
$$

The strain energy of the bending region in the beryllium bronze pieces is [19].

$$
U=\frac{R r \psi \theta}{2}\left[\Delta k_{x} \Delta k_{y}\right]\left[\begin{array}{l}
M_{x} \\
M_{y}
\end{array}\right] .
$$

From Eq. (3), we have

$$
U=\frac{R r \psi \theta}{2}\left[\begin{array}{ll}
\frac{1}{r} & \frac{1}{R}
\end{array}\right]\left[\begin{array}{c}
D \frac{1}{r}+D \mu \frac{1}{R} \\
D \mu \frac{1}{R}+D \frac{1}{r}
\end{array}\right] .
$$

The upper equations can be simplified as

$$
U=\frac{R \psi \theta}{2 r} D+\psi \theta D \mu+\frac{r \psi \theta}{2 \mathrm{R}} D .
$$

According to the principle of minimum potential energy, it follows from these equations that

$$
\frac{\partial U}{\partial r}=0
$$

Substituting Eq. (5) into Eq. (6),

$$
\frac{R \psi \theta}{2 r^{2}} D-\frac{\psi \theta}{2 R} D=0
$$



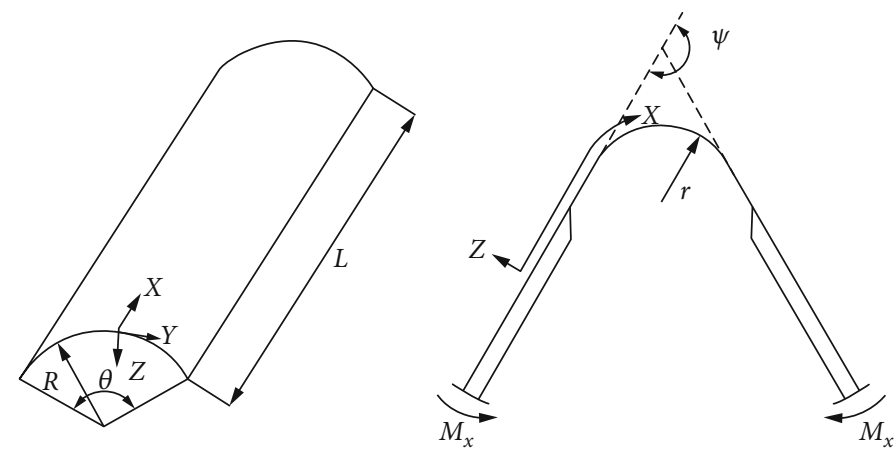

FIGURE 3: The curvature changes during the shell bending.

Solved from this equation:

$$
r=R
$$

Substituting Eq. (8) into Eq. (1) to obtain the following:

$$
M_{x}=D\left(\mp \frac{1}{R}+\mu \frac{1}{R}\right),
$$

where $\mp 1 / R$ are the curvature changes $\Delta k_{x}$ and $\Delta k_{y}$ when the beryllium bronze pieces are forward and reverse bent, respectively.

Because $M_{x}$ is the bending moment per unit arc length, there are

$$
\left.\begin{array}{l}
M_{+}=M_{x} \theta R=D \theta(1+\mu) \\
M_{-}=M_{x} \theta R=D \theta(-1+\mu)
\end{array}\right\},
$$

where $\theta$ is the central angle of the section, the unit of which is radian.

For beryllium bronze pieces with a thickness of $t=$ $0.13 \mathrm{~mm}$, the bending stiffness of the thin shell is $D=$ $2.496 \times 10^{-2} \mathrm{~N} \cdot \mathrm{m}$. When substituting this into Eq. (10), the forward deployed moment of beryllium bronze pieces is $M_{+}=0.052 \mathrm{~N} \cdot \mathrm{m}$, and the reverse deployed moment is $M_{-}=-0.052 \mathrm{~N} \cdot \mathrm{m}$.

3.2. Deployed Moment for Lenticular Booms. A lenticular boom is composed of two inverted beryllium bronze pieces. The deployed moment for lenticular booms is the sum of the absolute values of two beryllium bronze pieces:

$$
M^{\prime}=M_{+}+M_{-} \text {. }
$$

Similarly, for the reinforcement pieces with the thickness, $t=0.15 \mathrm{~mm}$, the bending stiffness is $D=3.846 \times 10^{-2} \mathrm{~N} \cdot \mathrm{m}$. Substituting this into Eq. (10), the forward deployed moment of beryllium bronze pieces is $M_{+}^{*}=0.0805 \mathrm{~N} \cdot \mathrm{m}$, and the reverse deployed moment is $M_{-}^{*}=-0.0805 \mathrm{~N} \cdot \mathrm{m}$.

For lenticular booms that include several reinforcement pieces, the deployed moment can be calculated by the following equation:

$$
M=M^{\prime}+m \times M_{+}^{*}+n \times M_{-}^{*}
$$

where $m$ and $n$ are the numbers of reinforcement pieces that are forward bent and reverse bent, respectively.

For the lenticular booms of a self-deployable sunshield, the number of upper and lower enhancement pieces is 2 and 6 , and the deployed moment is $M=0.7485 \mathrm{~N} \cdot \mathrm{m}$.

\section{Deployed Moment Analysis of Lenticular Booms}

4.1. Analysis Method of Deployed Moment. The deployed moment of lenticular booms can be numerically investigated by stowing and deployment process analysis. FEA explicit algorithms are used to simulate the stowing and deployment processes of lenticular booms in the Abaqus/Explicit software. This program can easily simulate nonlinear contact and some extreme discontinuity problems. The dynamic behaviors are calculated forward from the previous time steps instead of iterative solutions. The program provides a good parallel acceleration ratio and can effectively avoid the convergence difficulty caused by repeated iteration, which can easily occur when an implicit algorithm is used to solve complex dynamic problems.

The central difference method is applied in Abaqus/Explicit to integrate the dynamic equations over time. $\mathbf{x}_{0}, \dot{\mathbf{x}}_{0}$, and $\ddot{\mathbf{x}}_{\mathbf{0}}$ are the displacement, velocity, and acceleration, respectively, at time $t=0$. The analysis time domain $T$ is divided into $n$ steps, and the time step is $\Delta t=T / n$. The solution at time $t$ is known, and the solution at time $t+\Delta t$ needs to be calculated. The acceleration and velocity vectors can be expressed by the displacement vector with the central difference method as follows:

$$
\begin{aligned}
& \ddot{\mathbf{x}}_{t}=\frac{1}{\Delta t^{2}}\left(\mathbf{x}_{t-\Delta t}-2 \mathbf{x}_{t}+\mathbf{x}_{t+\Delta t}\right), \\
& \dot{\mathbf{x}}_{t}=\frac{1}{2 \Delta t}\left(-\mathbf{x}_{t-\Delta t}+\mathbf{x}_{t+\Delta t}\right) .
\end{aligned}
$$

The displacement solution $\mathbf{x}_{t+\Delta t}$ at time $t+\Delta t$ can be solved by the dynamic equation at time $t$ with the following equation. The dynamic equation of the lenticular booms is

$$
\mathbf{M} \ddot{\mathbf{x}}_{t}+\mathbf{C} \dot{\mathbf{x}}_{t}+\mathbf{K} \mathbf{x}_{t}=\mathbf{Q}_{t}
$$




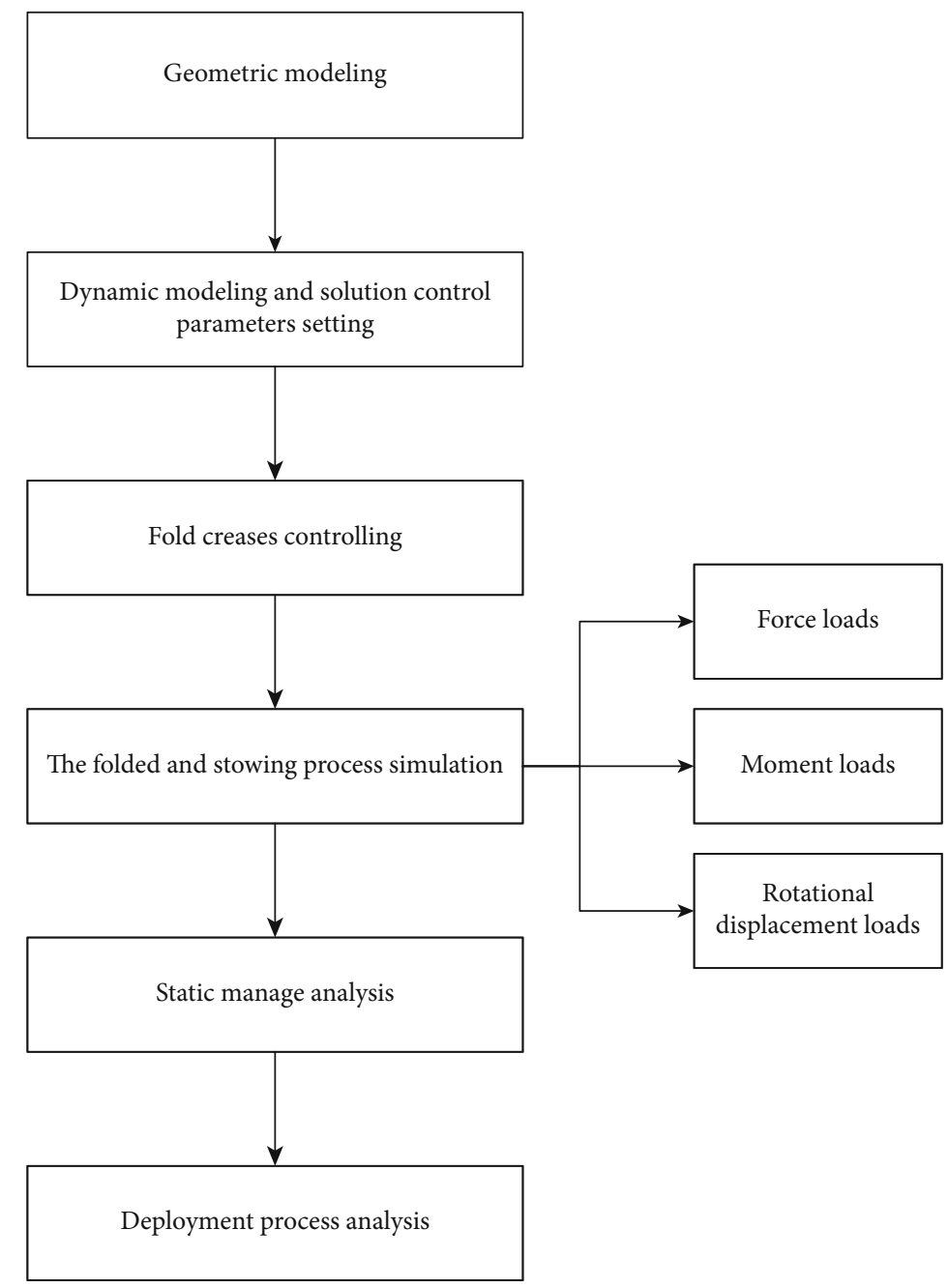

Figure 4: Flow chart of stowing and deployment process analysis.

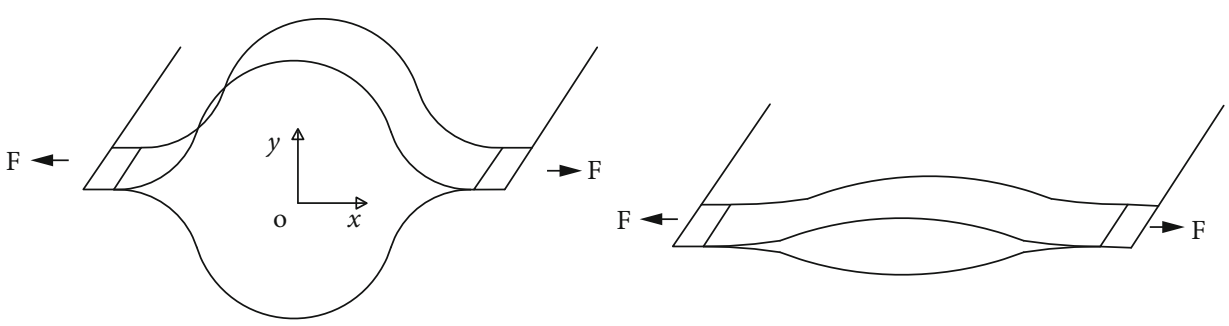

Figure 5: Pull flattening process of lenticular booms.

where $\mathbf{M}, \mathbf{C}$, and $\mathbf{K}$ are the mass, damping, and stiffness matrices, respectively, $\mathbf{x}_{t}$ is the position vector at time $t$, and $\mathbf{Q}_{t}$ is the external driving force.

Substituting Eq. (13) into Eq. (14) yields

$$
\begin{aligned}
\left(\frac{1}{\Delta t^{2}} \mathbf{M}+\frac{1}{2 \Delta t} \mathbf{C}\right) \mathbf{x}_{t+\Delta t}= & \mathbf{Q}_{t}-\left(\mathbf{K}-\frac{2}{\Delta t^{2}} \mathbf{M}\right) \mathbf{x}_{t} \\
& -\left(\frac{1}{\Delta t^{2}} \mathbf{M}-\frac{1}{2 \Delta t} \mathbf{C}\right) \mathbf{x}_{t-\Delta t}
\end{aligned}
$$

When the system deformations to the state $S_{i}$ at time $t$, the corresponding velocity, acceleration, and displacement at time $t+\Delta t$ are determined by Eq. (15) and Eq. (13). The state $S_{i}$ is updated to the next state $S_{i+1}$ at time $t+\Delta t$.

The initial state of the structures is a static state. Therefore, at time 0 , the initial values of displacement, velocity, and acceleration are

$$
\left.\mathbf{X}_{t}\right|_{t=0}=0,\left.\mathbf{X}_{t-\Delta t}\right|_{t=0}=0,\left.\frac{\partial \mathbf{X}_{t}}{\partial t}\right|_{t=0}=0,\left.\frac{\partial^{2} \mathbf{X}_{t}}{\partial t^{2}}\right|_{t=0}=0
$$




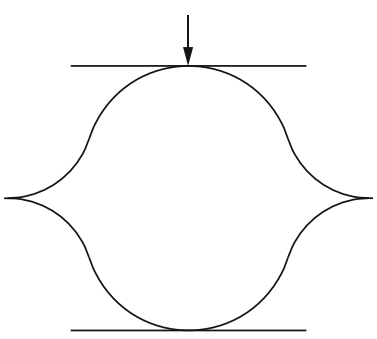

(a)

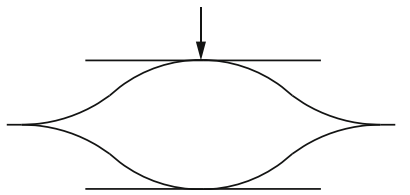

(b)

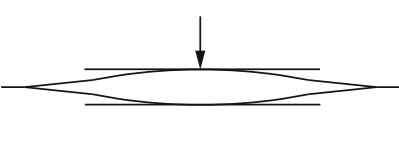

(c)

Figure 6: Pressure flattening process of lenticular booms.

The deployed moment of lenticular booms can be obtained by the stowing and deployment process dynamics analysis. The flow chart of the stowing and deployment process dynamics analysis is shown in Figure 4. First, the geometric models and dynamic model of the structures are established, and the control parameters of the FEA explicit solution are set. The dynamic analysis is divided into four steps: in the first step, fold creases are controlled by the pull flattening method or press flattening method. Then, the folded loads are applied on the structures to simulate the stowing process in the second step. The folded state can be determined after this step. The types of folded loads can be force loads, moment loads, and rotational displacement loads. Before the deployment process analysis, a static management analysis must be conducted to eliminate the subsequent effects of the stowing process. Finally, the deployment process analysis is conducted when the structures are subjected to the actual loads and constraint conditions.

\subsection{Control Methods for Fold Crease}

4.2.1. Pull Flattening Method. The pull flattening process of lenticular booms is shown in Figure 5. The working state of the lenticular booms after full deployment is a lenticular section. The stowing and deployment processes in the pull flattening method mainly include two tension forces applied on both side nodes at the fold crease section. The upper and lower beryllium bronze pieces elastically deform until the sections become flat and contact each other. Thus, the out-of-plane stiffness of the lenticular booms in this section is minimal. Then, the lenticular booms are folded and constrained. When the constraint is removed, the lenticular booms are deployed by releasing the strain energy. When the fold crease section changes back into a lenticular section, the lenticular booms achieve a fully deployed state.

4.2.2. Press Flattening Method. The press flattening process of lenticular booms is shown in Figure 6. The initial section shape of the lenticular booms is shown in Figure 6(a). The stowing and deployment processes in the press flattening method mainly include the following: first, vertical pressure is applied on the lenticular booms at the fold crease section, as shown in Figure 6(b). The contact between the upper and lower beryllium bronze pieces gradually expands from a point to a line, and the upper and lower pieces of the lenticular booms are com-

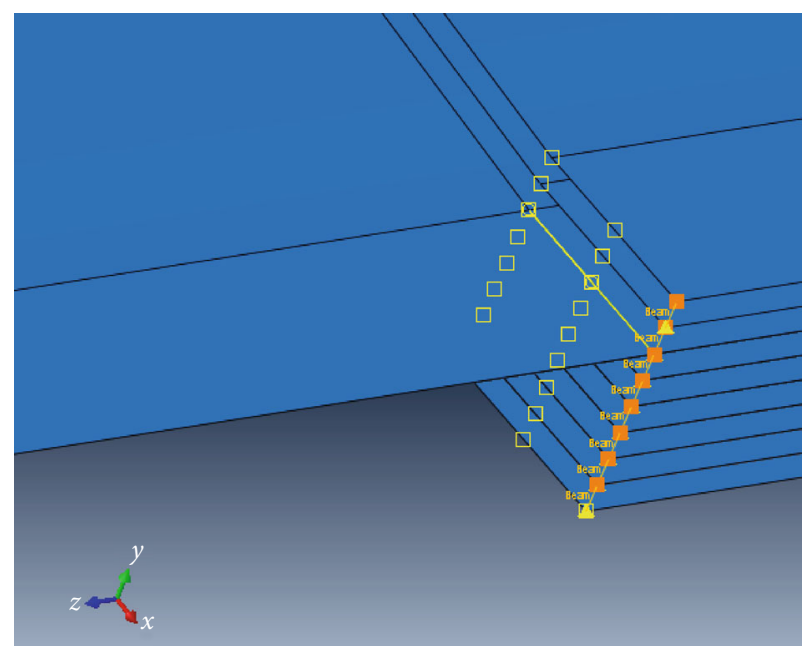

FIgURE 7: Constraint simulation of $3 \mathrm{M}$ tapes.

pressed into a flat shape. The out-of-plane stiffness of the lenticular booms in the section is minimal, as shown in Figure 6(c). Then, the lenticular booms are folded and constrained. Finally, after the constraint is released, the lenticular booms are self-deployed to the initial configuration by releasing the strain energy.

4.3. Dynamic Modeling. The dynamic finite element analysis model of the lenticular booms is established in the Abaqus software. First, geometric models of the single beryllium bronze piece and the reinforcing piece in the pod rod are established. Then, the lenticular boom model is obtained by assembling two beryllium bronze pieces and eight reinforcing pieces.

The edge connection between the upper and lower beryllium bronze pieces is set as follows: the pieces are independent of each other in the length range of $0 \mathrm{~mm}$ $250 \mathrm{~mm}$ and welding connection in the length range of $250 \mathrm{~mm}-610 \mathrm{~mm}$. Two $1 \mathrm{~mm}$ wide areas in the left and right edges are connected by binding ( ${ }^{*}$ tie) to simulate this welding connection.

There are 1 contact pair between the upper and lower beryllium bronze pieces, 2 contact pairs between reinforcing pieces and beryllium bronze, and 6 contact pairs between adjacent reinforcing pieces. These contact pairs are established by ${ }^{*}$ surface-to-surface contact. The corresponding contact algorithms are set. The "hard contact" model is adopted for the normal 


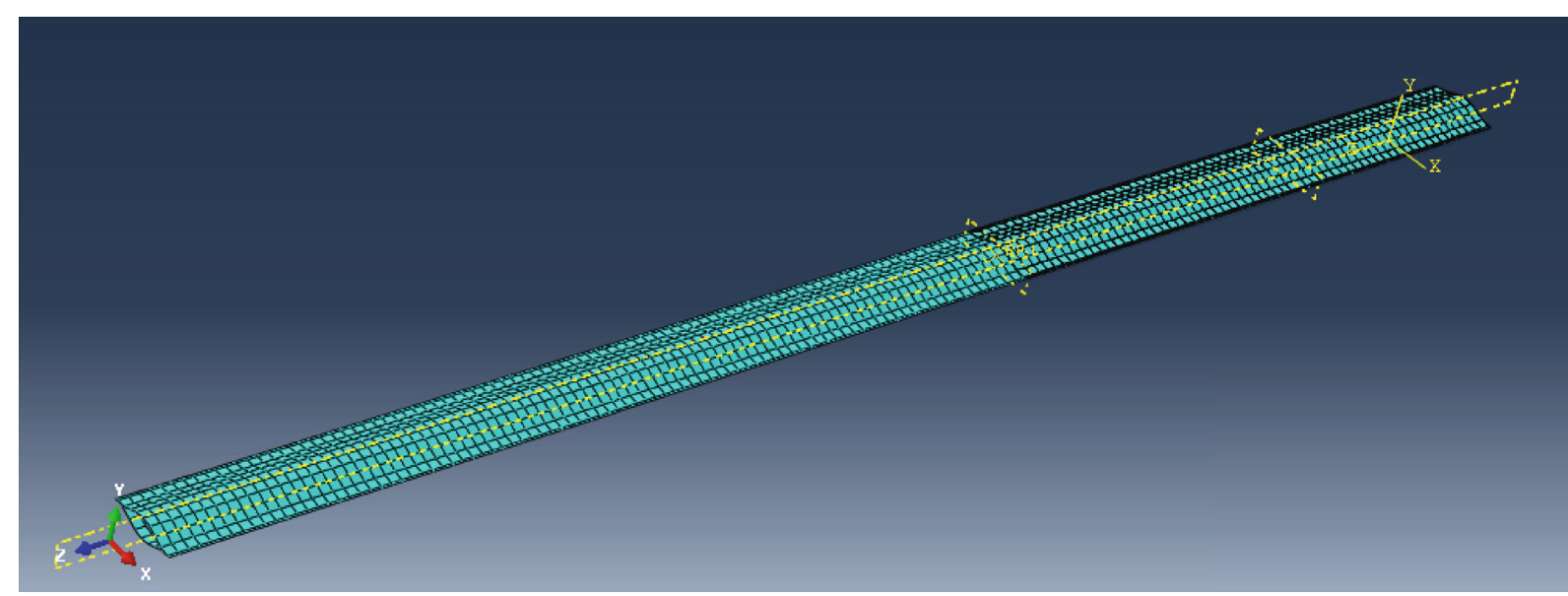

FIgURE 8: FEA model of the lenticular booms.

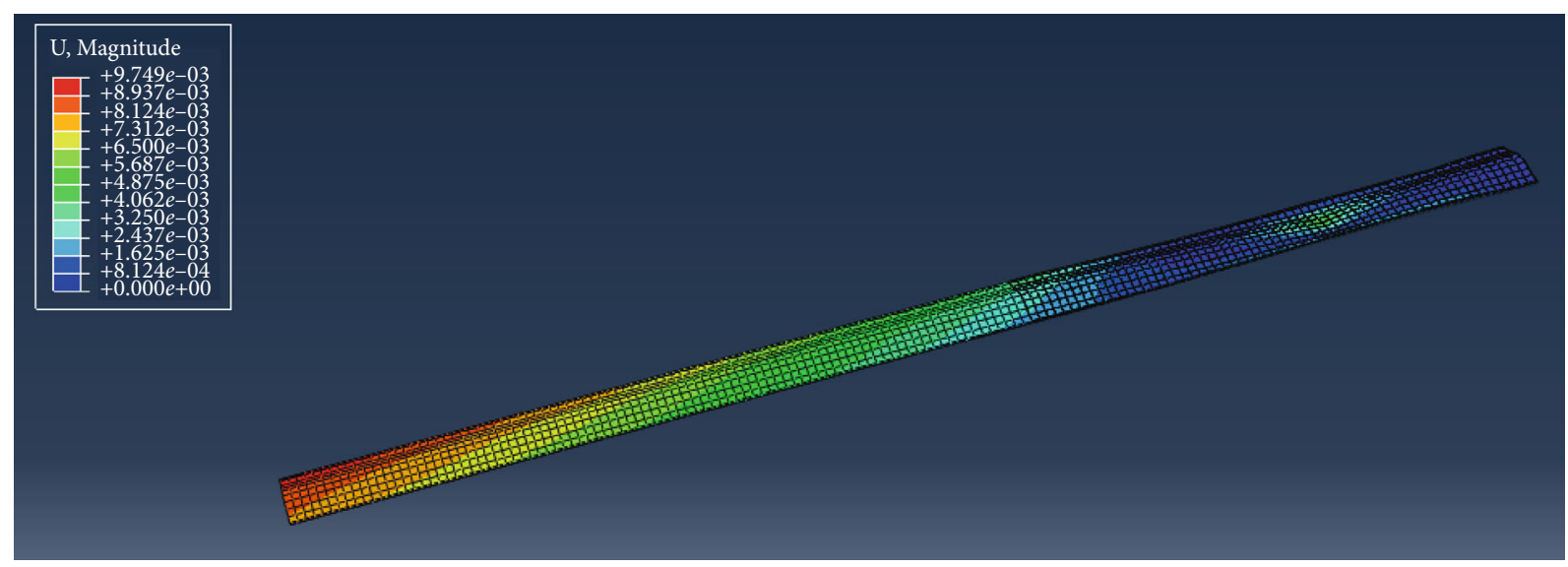

(a) Displacement

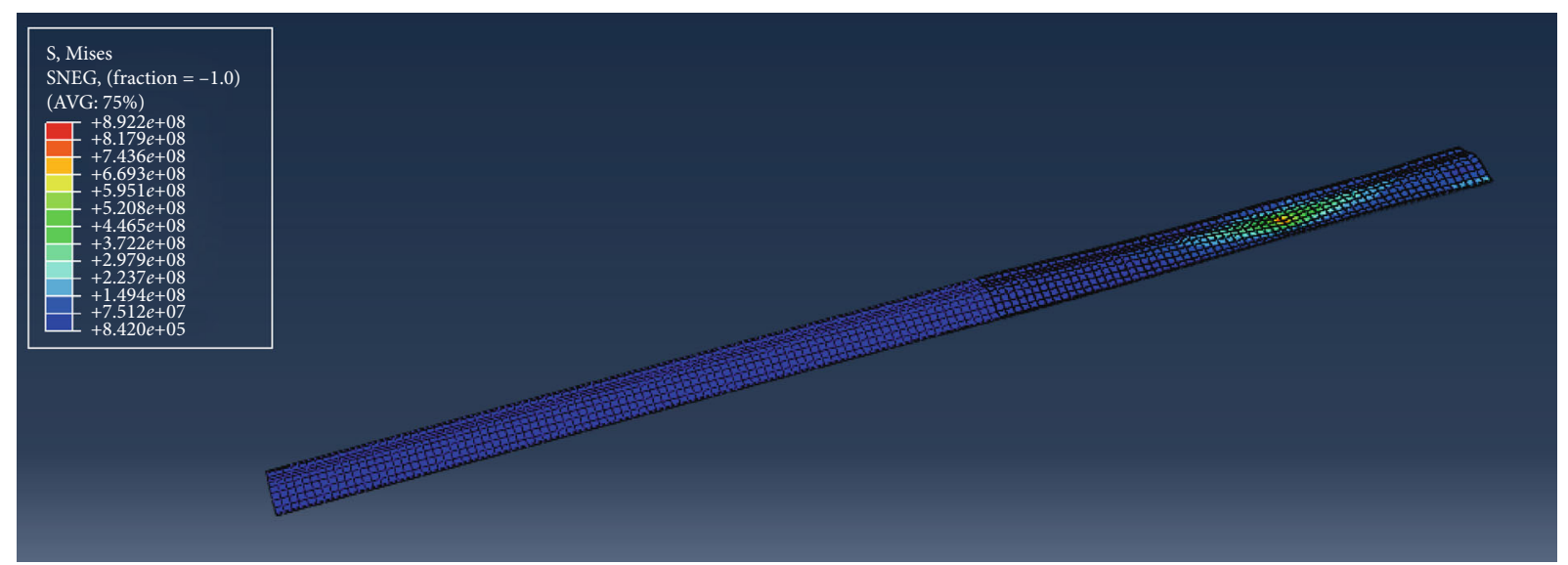

(b) Stress distribution

FIGURE 9: Configuration after the press flattening process.

contact, the penalty function method is used for the constraint execution, and the tangential friction behavior is ignored.

The section $100 \mathrm{~mm}$ from the fixed end of the lenticular booms is defined as section S1. The section $250 \mathrm{~mm}$ from the fixed end is defined as section S2. The center node of section S2 was established as a reference point. All the nodes on this section were coupled with the reference point by using * kinematic coupling so that all the nodes will move together with the reference point. The reference point was used as the load-applying node in the subsequent dynamic analysis process.

At the free end of the reinforcing pieces, connection elements (*connection) between the reinforcing pieces and the beryllium bronze pieces, as well as between the adjacent 


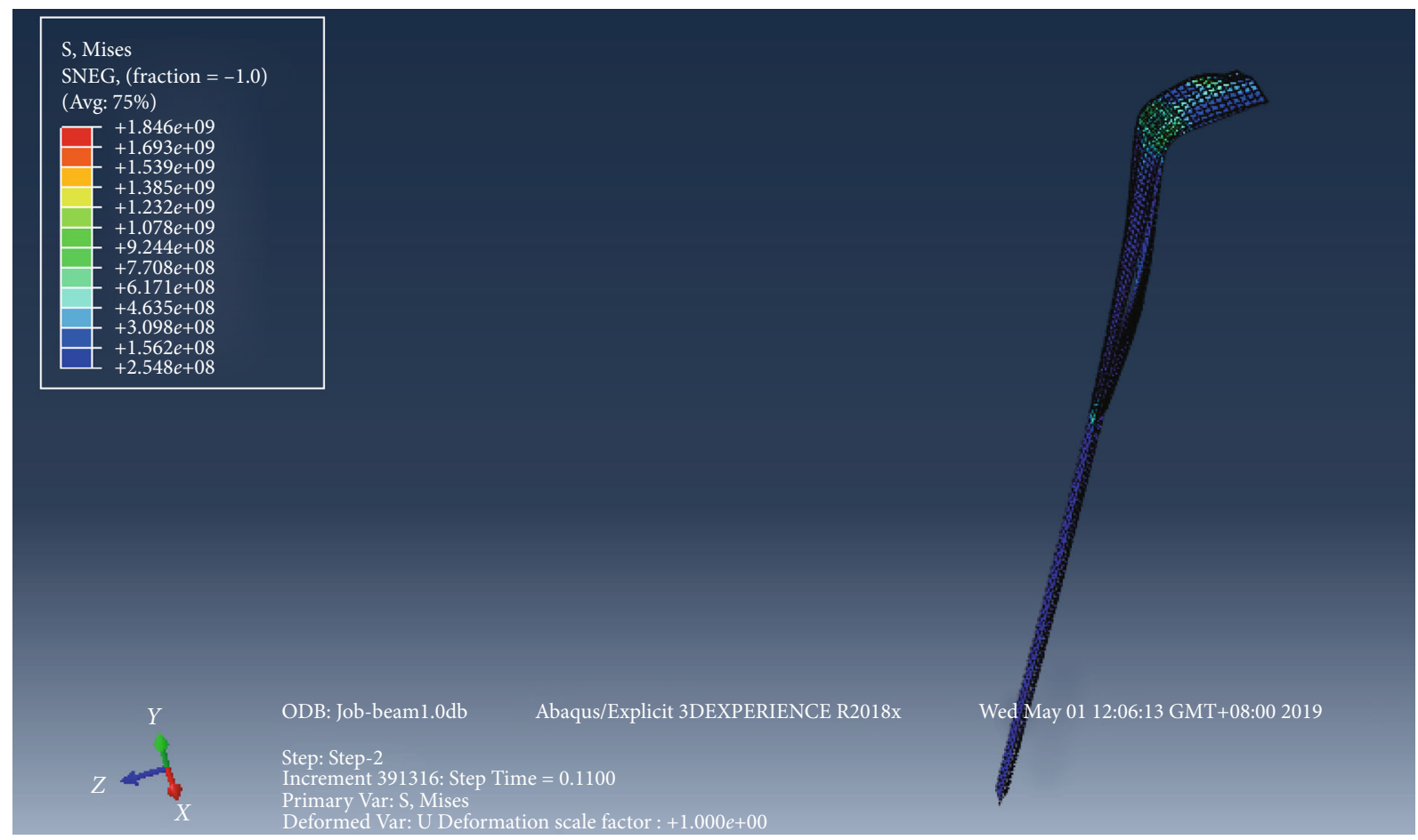

Figure 10: Configuration and stress of the folded state.
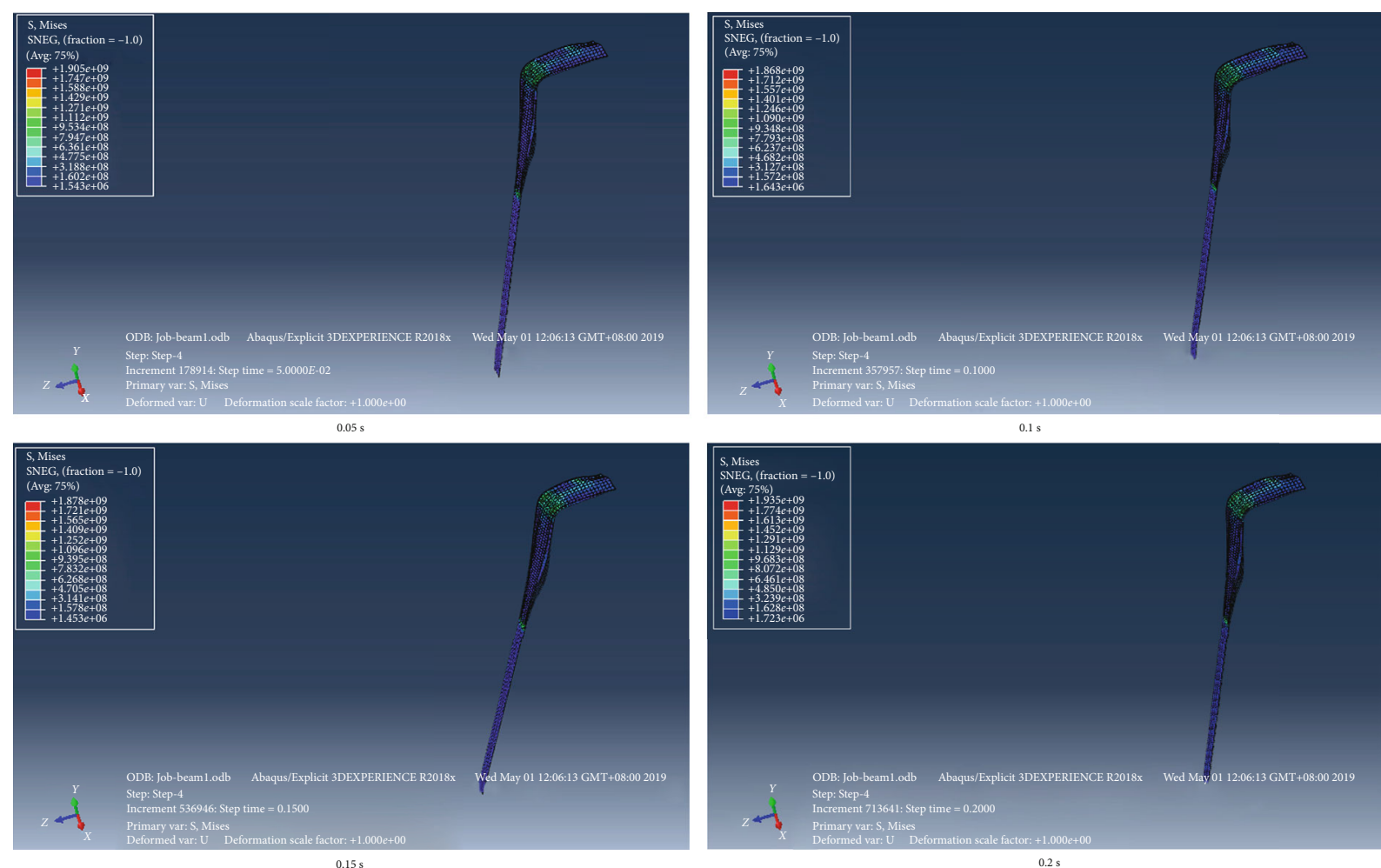

Figure 11: Dynamic behaviors of the lenticular booms.

reinforcing pieces, were established by means of *beam to simulate the constraint of $3 \mathrm{M}$ tapes between the reinforcing pieces and the beryllium bronze pieces, as shown in Figure 7.
The boundary conditions of the lenticular booms are set, with the right end fixed and the other end free. To suppress the low-frequency vibration after full deployment, the Rayleigh damping of the beryllium bronze material is set. 


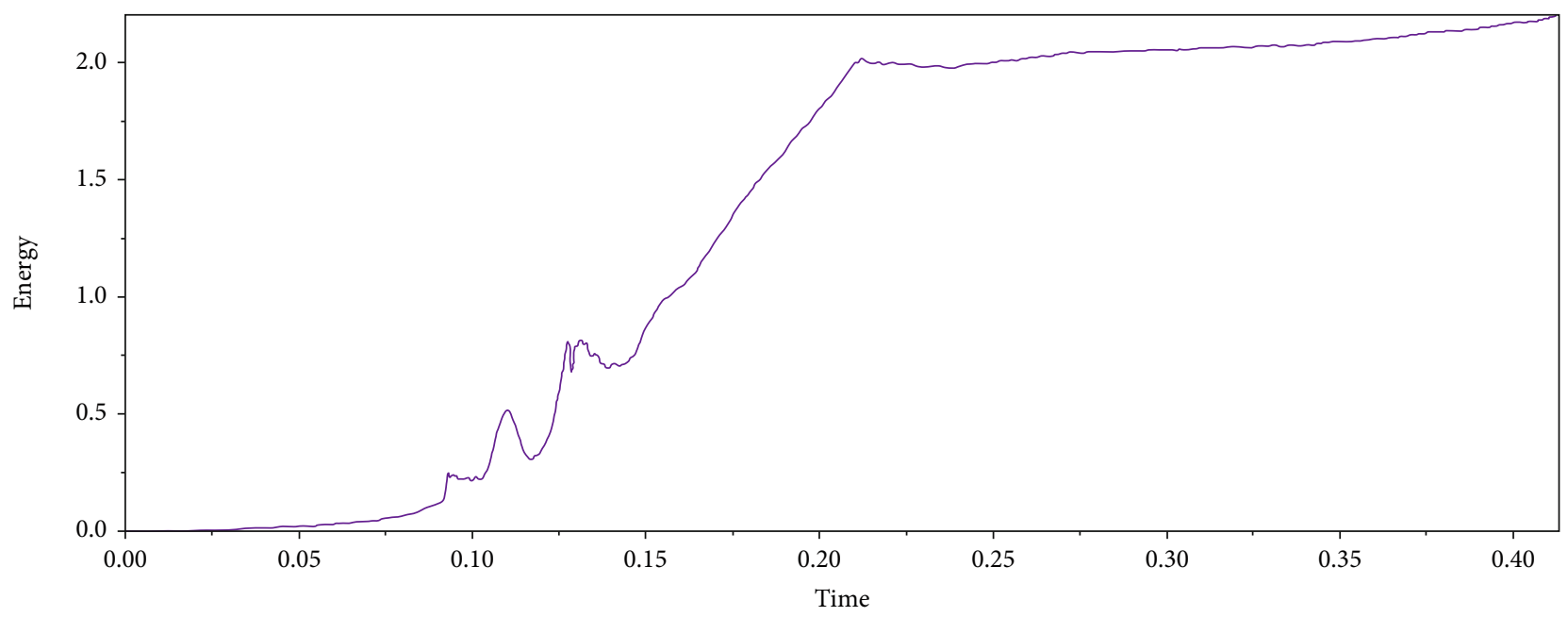

_ ALLSE whole model

FIGURE 12: Time history of elastic potential energy.

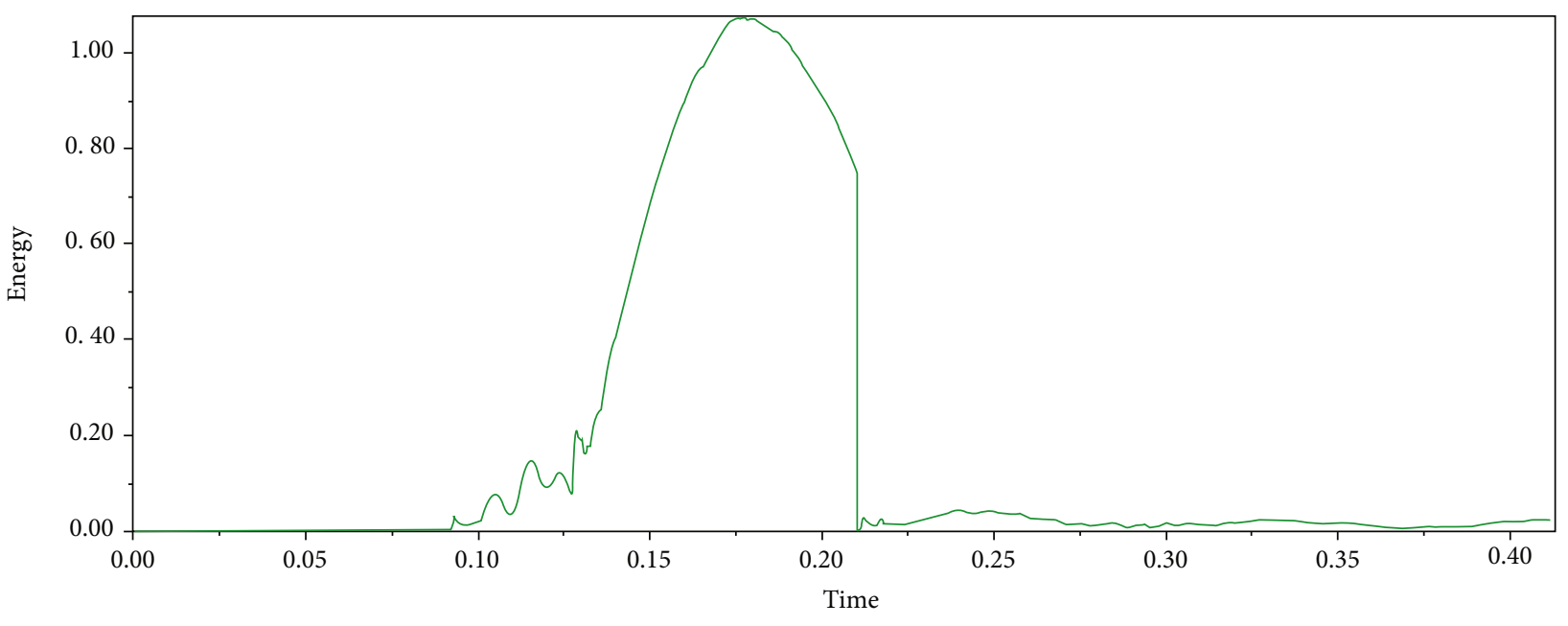

Figure 13: Time history of kinetic energy.

The geometric model is meshed to obtain the dynamic FEA model, as shown in Figure 8.

To analyze the deployed moment of the lenticular booms, four analysis steps ( ${ }^{*}$ step) are set as follows: step 1 simulates the flattening process of lenticular booms in fold crease section S1. Step 2 simulates the folding and stowing processes; step 3 simulates the static management processing to eliminate the subsequent effects of the stowing process; step 4 simulates the dynamic behaviors of the lenticular booms after a weight was suspended at section S2 to obtain the deployed moment.

In the simulation of the press flattening process, a $70 \mathrm{~N}$ force pair of $Y$ axis directions is applied to the vertexes at section $\mathrm{S} 1$ for the top and bottom reinforcement pieces. In the simulation of the folding and stowing processes, a $30 \mathrm{~N}$ force in the $-Y$ axis direction is applied to the reference point, which is defined previously. In step 4 , a $5.5 \mathrm{~N}$ force in the $-Z$ axis direction is also applied to the reference point to simulate the load of the suspended weight.
As a rigid-flexible multibody nonlinear system, the stowing and deployment processes of lenticular booms are very complex. The nonlinear dynamic characteristics of geometry and contact are highlighted. Therefore, parallel computation and acceleration strategies are needed to complete the simulation of the whole process. In the solver setting, full analysis is selected for the job type, and 4 processors are set for parallel computation.

4.4. Analysis Results of Deployed Moment. The displacement and stress distribution of the lenticular booms after the press flattening process are shown in Figure 9. The upper and lower beryllium bronze pieces are compressed into a flat shape under press forces, while the free end of the lenticular booms is deformed upwards. Therefore, the out-of-plane stiffness of the lenticular booms in section S1 becomes small. The stress near the point of the press force is greatest, and the stresses in other areas are small. 


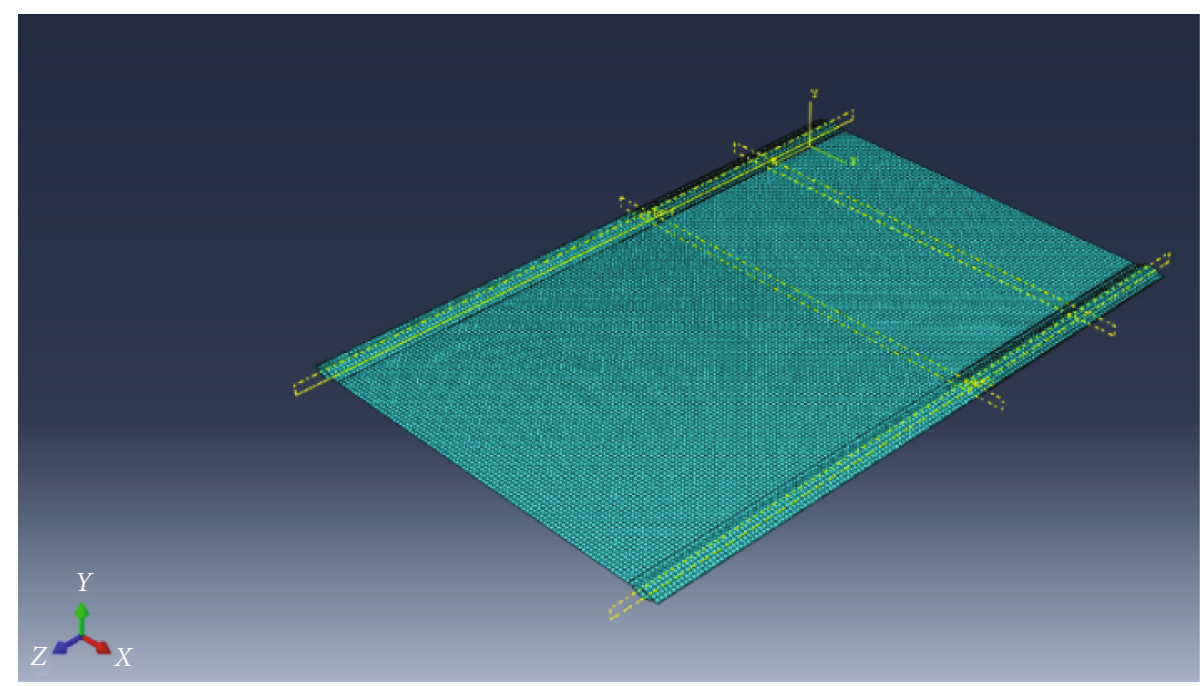

FIGURE 14: FEA model of the self-deployable sunshield.

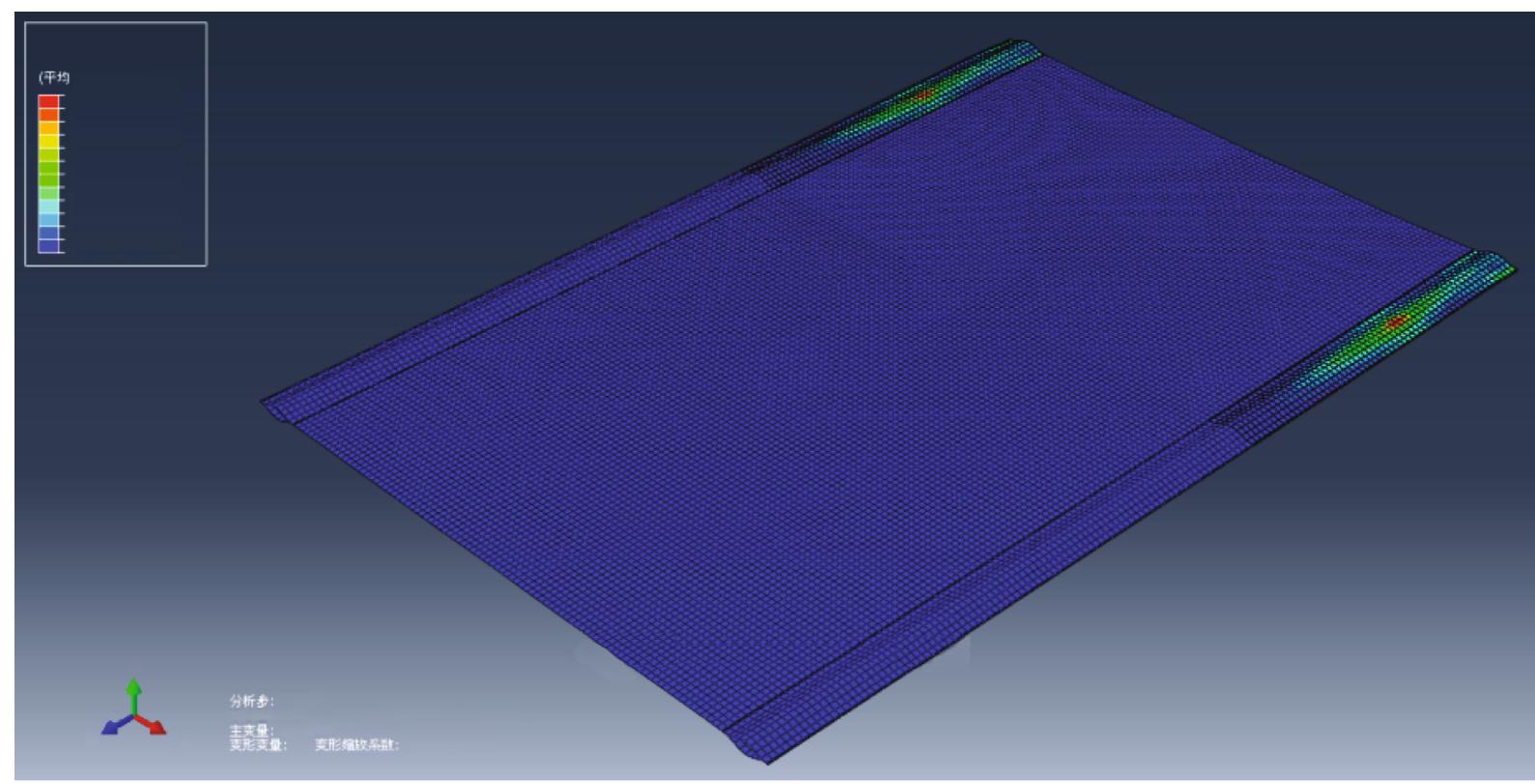

Figure 15: Configuration after the press flattening process.

During the folding process, after the nodal force is applied at the reference point of section S2, the lenticular booms are folded. The configuration and stress distribution of the folded state are shown in Figure 10. The deformation in the free end is largest, and the fold crease of the lenticular booms is near section S1. After the folding process, the folding angle is close to $90^{\circ}$. This indicates that the press flattening method can be used effectively for controlling the fold crease. There are large stresses near sections S1 and S2 and the fixed end. The stresses in other areas are small.

In the deployed moment analysis, the dynamic behaviors of the lenticular booms are shown in Figure 11 after applying a folded force at the reference point, the value of which is continuously adjusted. According to the simulation results, after applying the folded force $5.4 \mathrm{~N}$ at the reference point, the lenticular booms only fluctuate slightly and maintain the equilibrium state. Then, the deployed moment of the lenticular booms is equal to the moment from the folded force. It can be seen that the deployed moment of the lenticular booms is $M=5.4 \times 0.15=0.81 \mathrm{~N} \cdot \mathrm{m}$, which is close to the theoretical result $0.748 \mathrm{~N} \cdot \mathrm{m}$ obtained in Section 3.2, with an error between the two results of $7.6 \%$. Because that the interactions between multiple beryllium bronze pieces do not be considered in the theoretical model, this error is acceptable.

The time history of elastic potential energy and kinetic energy in the whole process is shown in Figures 12 and 13. The time history curves of the energy, elastic potential energy, and kinetic energy change slowly during the press flattening process. In the folded process, the elastic potential energy increases significantly, and the kinetic energy increases at first and then decreases. The kinetic energy 


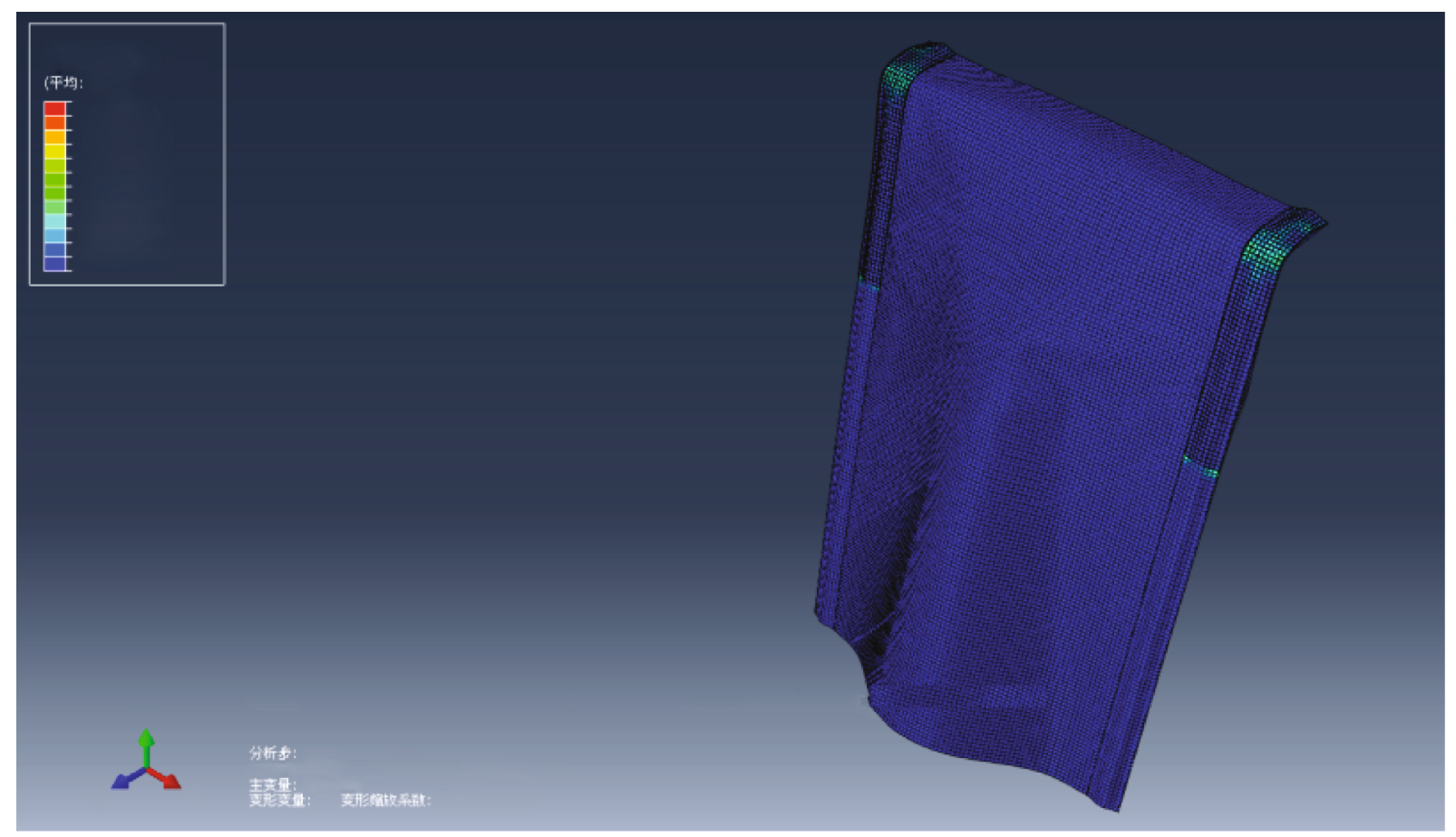

FIGURE 16: Configuration and stress distribution of the folded state.

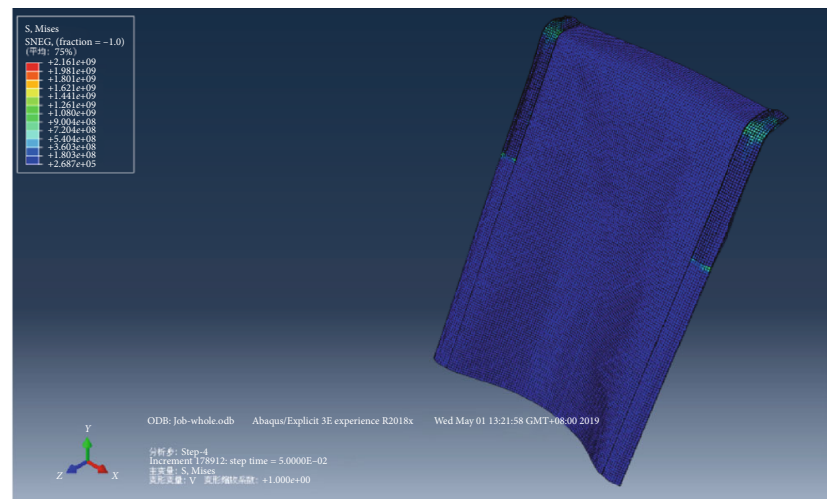

$0.05 \mathrm{~s}$

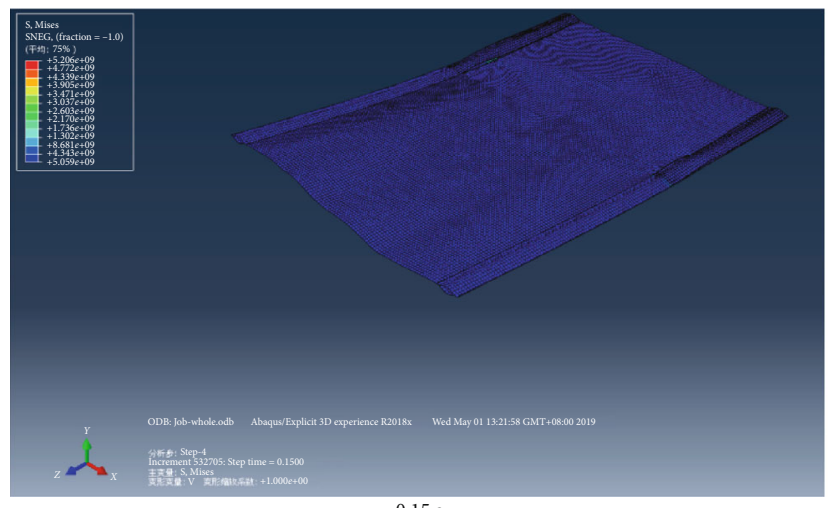

$0.15 \mathrm{~s}$

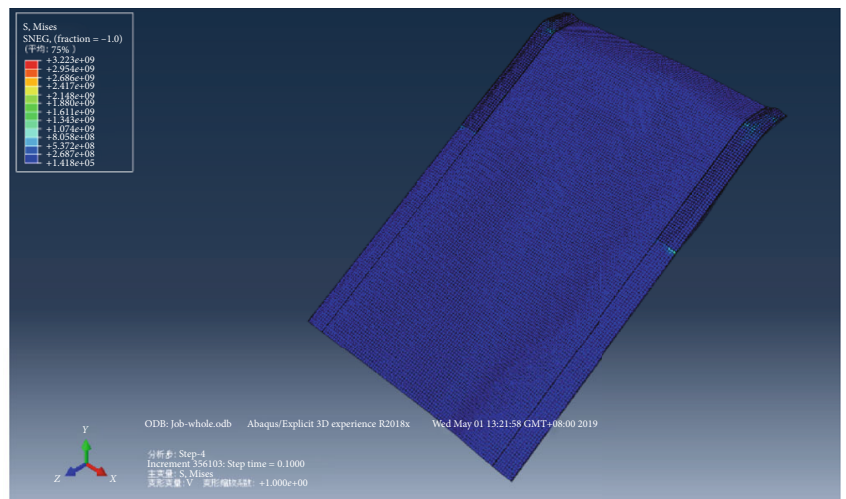

$0.1 \mathrm{~s}$

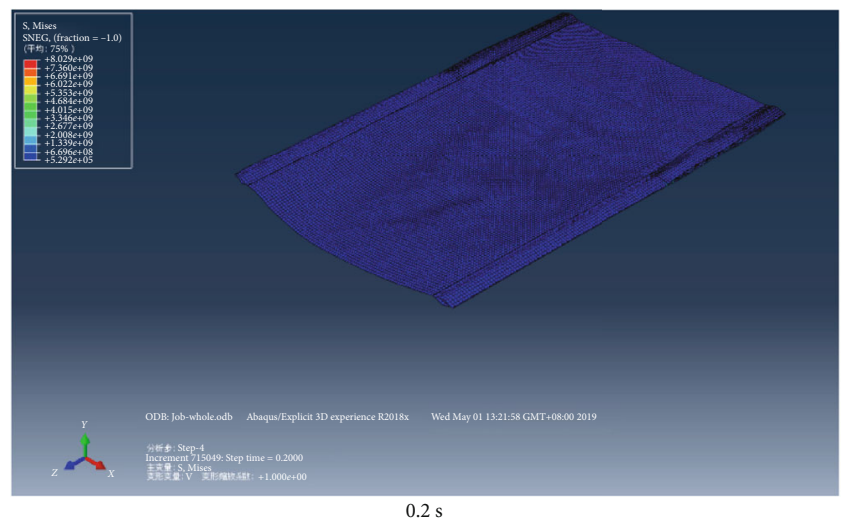

$0.2 \mathrm{~s}$

FIGURE 17: Dynamic behaviors of self-deployable sunshields.

reduces to zero after static management processing, and the elastic potential energy maintains the same value. In the subsequent analysis, the kinetic energy oscillates to 0 , and the elastic potential energy basically remains unchanged. It can also be seen from the energy time history that the equilibrium state has been reached after applying the folded force $5.4 \mathrm{~N}$. 


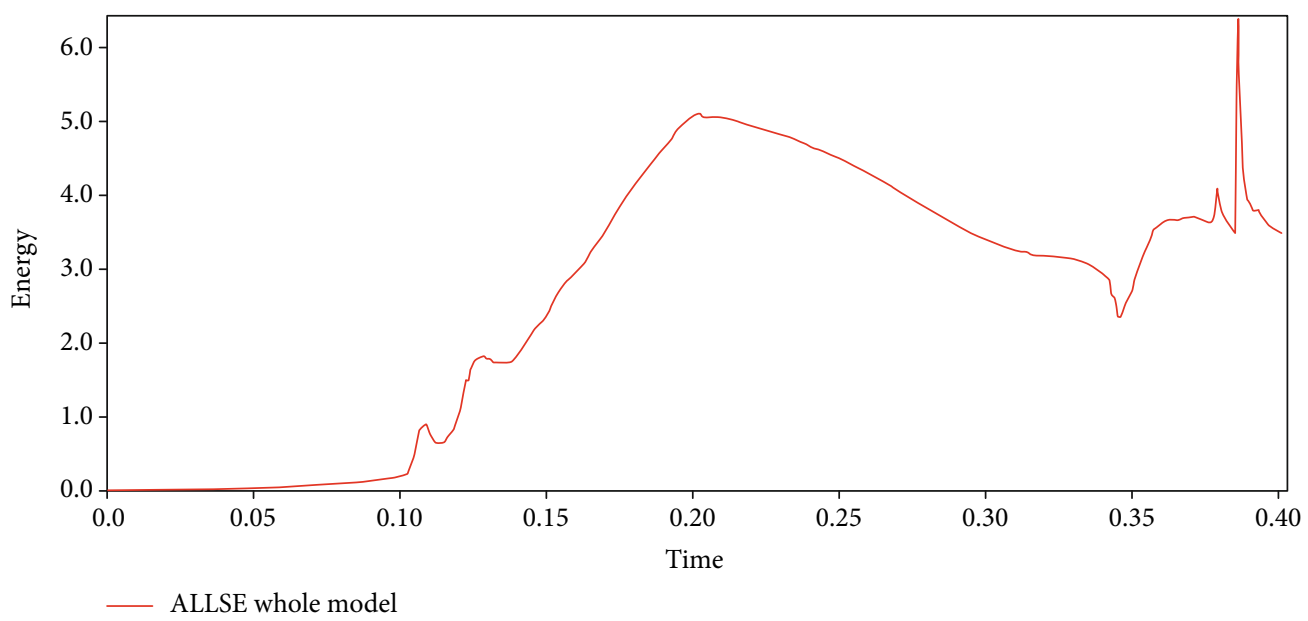

FIGURE 18: Time history of elastic potential energy.

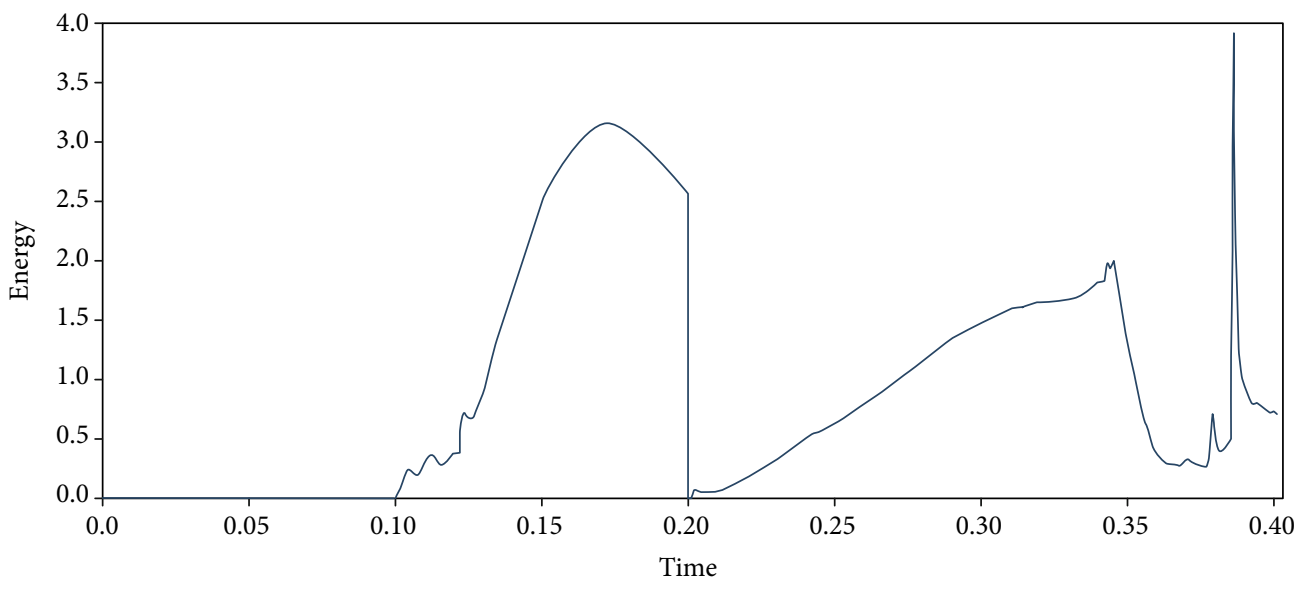

Figure 19: Time history of kinetic energy.

The work done by the folded force is first converted into kinetic energy and elastic potential energy. Finally, it is stored in the lenticular booms in the form of elastic potential energy.

\section{Stowing and Deployment Process Simulation of Self-Deployable Sunshield}

The self-deployable sunshield includes two lenticular booms and skin structures. The dynamic FEA model of lenticular booms is described in detail in Section 4.3. After the geometric model of the skin structures was established, the geometric model of the self-deployable sunshield can be assembled. Two long edges of the skin structures and the edges of two lenticular booms are connected by using a binding (*tie) to simulate the sleeve connection here. The dynamic FEA model of the self-deployable sunshield is shown in Figure 14.

The configuration and stress distribution of the selfdeployable sunshields after the press flattening process at the section $100 \mathrm{~mm}$ from the fixed end are shown in Figure 15. The stress is mainly distributed near the point of the press force in two lenticular booms, and there is little stress on other areas of two lenticular booms and skin structures. The deformation of the structures is small except the areas near the point of the press force.

After the $40 \mathrm{~N}$ force is applied to the reference point during the folding process, the self-deployable sunshield has undergone a large deformation. The configuration and stress distribution of the folded state are shown in Figure 16. The skin structure is folded while the lenticular booms are folded. After the folding process, the folding angle is close to $90^{\circ}$. There are large stresses near sections S1 and S2 and the fixed ends of the two lenticular booms. The stresses in other areas of the two lenticular booms are small. The stresses near the fold crease on the skin structures are still small. It can also be seen that the bending stiffness of the skin structure is very small.

During the deployment process analysis, all constraints and loads need to be released except the fixed end of two lenticular booms. The dynamic behaviors of self-deployable sunshields are shown in Figure 17. The analysis results show that the sunshield can be self-deployed smoothly by releasing the strain energy in two lenticular booms. The skin structure 
is pulled by two lenticular booms and begins to deploy. No other driving force is required for the entire deployment process. Finally, the lenticular booms and skin structure are deployed to a nearly straight state.

The time history curves of elastic potential energy and kinetic energy during the whole stowing and deployment processes are shown in Figures 18 and 19. It can be seen from these curves that the elastic potential energy and kinetic energy change slowly during the press flattening process. In the folded process, the elastic potential energy increases significantly, and the kinetic energy increases at first and then decreases. The kinetic energy reduces to zero after static management processing, and the elastic potential energy maintains the same value. The work done by the folded force is first converted into kinetic energy and elastic potential energy. Then, it is stored in the lenticular booms in the form of elastic potential energy. In the deployment process, the kinetic energy increases and the elastic potential energy decreases slowly. Finally, the kinetic energy oscillates to 0 , and the sunshields are fully deployed.

\section{Conclusion}

In the paper, the deployed moment of the lenticular booms is evaluated by theoretical model and numerical simulation model. The deployed moment of the lenticular booms obtained from numerical simulations is $0.81 \mathrm{~N} \cdot \mathrm{m}$, which is close to the theoretical result of $0.748 \mathrm{~N} \cdot \mathrm{m}$. The error between the two results is $7.6 \%$. The analysis results also indicate that the press flattening method can be used effectively for controlling the fold crease. An explicit analysis method for stowing and deployment processes of the selfdeployable sunshields is proposed, and the dynamic responses are obtained. There are large stresses near sections S1 and S2 and the fixed end of the two lenticular booms in the folded state. The stresses near the fold crease on the skin structures are small. The analysis results show that the sunshield can be self-deployed smoothly by releasing the strain energy in two lenticular booms.

However, because the reinforcement pieces are considered in the dynamic model, the calculation cost of contact collision between various parts is very large, and divergence can easily occur during the analysis. In subsequent research, the model of reinforcement piece needs to be simplified. The relevant experimental studies of the lenticular booms will be carried out in the future.

\section{Data Availability}

The data used to support the findings of this study are available from the corresponding author upon request.

\section{Conflicts of Interest}

The authors declare that they have no conflicts of interest.

\section{Acknowledgments}

This work was supported by the National Natural Science Foundation of China (Grant Nos. 91748209 and 11402229), the Natural Science Foundation of Zhejiang Province (Grant No. LY17A020003), and the Fundamental Research Funds for the Central Universities (Grant Nos. 2018QNA4054 and 2019QNA4057).

\section{References}

[1] J. D. Johnston, B. D. Ross, J. Blandino, and J. Lawrence, "Development of sunshield structures for large space telescope," Proceedings of the SPIE, vol. 4850, pp. 209-220, 2003.

[2] M. Clampin, "The James Webb space telescope (JWST)," Advances in Space Research, vol. 41, no. 12, pp. 1983-1991, 2008.

[3] R. A. Franck, "Thermal design, build, and test of the JWST aft optics subsystem," Cryogenics, vol. 64, pp. 235-239, 2014.

[4] D. W. Robinson and R. S. McClelland, "Mechanical overview of the international X-Ray observatory," in 2009 IEEE Aerospace conference, pp. 1-10, Big Sky, MT, USA, 2009.

[5] G. Pica, L. Ciofaniello, and S. Mattei, "High-resolution deployable telescope for satellite applications," in Proceedings Volume 5234, Sensors, Systems, and Next-Generation Satellites VII, pp. 1-8, Barcelona, Spain, 2003.

[6] D. Michael, A. Kirk, and K. Bernard, "Design and development of an in-space deployable sun shield for Atlas Centaur," in AIAA SPACE 2008 Conference \& Exposition, pp. 1-11, San Diego, California, 2008.

[7] O. Allam, K. Draiche, A. A. Bousahla et al., "A generalized 4unknown refined theory for bending and free vibration analysis of laminated composite and sandwich plates and shells," Computers and Concrete, vol. 26, no. 2, pp. 185-201, 2020.

[8] M. A. Khadimallah, M. Hussain, and K. M. Khedher, "Backward and forward rotating of FG ring support cylindrical shells," Steel and Composite Structures, vol. 37, no. 2, pp. 137-150, 2020.

[9] M. Hussain, M. N. Naeem, M. S. Khan, and A. Tounsi, "Computer-aided approach for modelling of FG cylindrical shell sandwich with ring supports," Computers and Concrete, vol. 25, no. 5, pp. 411-425, 2020.

[10] T. Cuong-le, K. D. Nguyen, N. Nguyen-Trong, S. Khatir, H. Nguyen-Xuan, and M. Abdel-Wahab, "A threedimensional solution for free vibration and buckling of annular plate, conical, cylinder and cylindrical shell of FG porouscellular materials using IGA," Composite Structures, vol. 259, no. article 113216, 2020.

[11] M. S. H. Al-Furjan, M. Habibi, D. W. Jung et al., "A computational framework for propagated waves in a sandwich doubly curved nanocomposite panel," Engineering with Computers, vol. 36, 2020.

[12] K. Behrouz, J. Maziar, and T. Abdelouahed, "On pre-stressed functionally graded anisotropic nanoshell in magnetic field," Journal of the Brazilian Society of Mechanical Sciences and Engineering, vol. 41, article 495, 2019.

[13] M. Leipold, H. Runge, and C. Sickinger, "Large SAR membrane antennas with light weight deployable booms," in 28th ESA Antenna Workshop on Space Antenna Systems and Technologies, ESA/ESTEC, pp. 1-8, Noordwijk, Netherlands, 2005. 
[14] C. Sickinger, L. Herbeck, and E. Breitbach, "Structural engineering on deployable CFRP booms for a solar propelled sailcraft," Acta Astronautica, vol. 58, no. 4, pp. 185-196, 2006.

[15] N. Katsumata, M. C. Natori, and H. Yamakawa, "Analysis of dynamic behavior of inflatable booms in zigzag and modified zigzag folding patterns," Acta Astronautica, vol. 93, no. 1, pp. 45-54, 2014.

[16] X. Yan, Z. Yao, G. Fuling, H. He, and H. Xian, "Parametric model method and deployment simulation of inflatable antenna structures," Journal of Aerospace Technology and Management, vol. 7, no. 2, pp. 219-230, 2015.

[17] Y. Yu and Y. Z. Luo, "Motion analysis of deployable structures based on the rod hinge element by the finite particle method," Proceedings of the Institution of Mechanical Engineers, Part G: Journal of Aerospace Engineering, vol. 223, pp. 955-965, 2009.

[18] J. Quirant, F. Cevaer, and S. Morterolle, "Conceptual design and analysis of a deployable structure with flexible joints," Journal of Aerospace Engineering, vol. 24, no. 3, pp. 277-284, 2011.

[19] K. A. Seffen and S. Pellegrino, "Deployment dynamics of tape springs," Proceedings of the Royal Society of London. Series A: Mathematical, Physical and Engineering Sciences, vol. 455, no. 1983, pp. 1003-1048, 1999.

[20] K. Kwok and S. Pellegrino, "Folding, stowage, and deployment of viscoelastic tape springs," AIAA Journal, vol. 51, no. 8, pp. 1908-1918, 2013. 